\section{§43. On Dust Dynamics in Tokamak Edge Plasmas}

Krasheninnikov, S. (UCSD),

Tomita, Y.,

Smirnov, R. (Grad. Univ. Adv. Studies),

Janev, R. (Macedonian Academy of Sciences and Arts)

We consider some aspects of dust dynamics in tokamak plasmas. We assume that dust density in a tokamak plasma is rather small; consequently, our consideration will be based on a single dust particle motion. Therefore, we shall ignore the collective phenomena associated with the dust. The plasma parameters in tokamak edge are very inhomogeneous, and absolute numbers of plasma density and temperature depend on discharge characteristics and on confinement regime. However, in order to simplify our estimates we will assume that electron, $T_{\mathrm{e}}$, and ion, $T_{\mathrm{i}}$, temperatures are close to $T_{\mathrm{e}}$ $\sim T_{\mathrm{e}} \sim 10 \mathrm{eV}$ and plasma density is about $3 \times 10^{13} \mathrm{~cm}^{-3}$. These values are typical for tokamak divertor plasmas.

We will show that in a tokamak dust can be very mobile, and during the duration of one shot dust particles can move through the edge plasma on distances that are much larger than the scrape-of-layer (SOL) width and comparable to tokamak radii.

While in perpendicular direction to a wall the friction force can be balanced by the electric force, in the directions along the wall the electric field is only due to wall and plasma inhomogeneities, but the friction force remains strong due to plasma flow along oblique magnetic field lines and ion diamagnetic and $E \times B$ flow in parallel direction to a wall. Within the sheath, the ion flow velocities of both parallel direction to a wall is close to ion flow velocity $V_{\mathrm{i}}$, which gives the following estimate for the $\mathrm{z}$ (parallel to magnetic field) and $x$ (perpendicular to magnetic field) components of the friction force $F_{\text {fric }}^{(z)} \sim F_{\text {fric }}^{(x)} \sim F_{\text {fric }}^{(s h)}$. At distances from the wall larger than $\rho_{\mathrm{i}}$, the diamagnetic ion flow velocity and, consequently, $F_{\text {fric }}^{(x)}$ significantly decrease, while the component $F_{\text {fric }}^{(z)}$ remains strong up to the distance $\alpha \lambda_{i N}$ from the wall, where $\lambda_{i N}$ is the mean-free-path of ion-neutral collisions. The importance of plasma diamagnetic and $E \times B$ flows on dust particle dynamics in low pressure weakly ionized plasmas was discussed in [17, 18]. However, in tokamak edge plasmas, the effect of these forces is much stronger. The unbalanced force will cause acceleration and motion of dust particle along the wall in both toroidal and radial directions. Since the force is proportional to the plasma pressure such acceleration is particularly strong within some toroidally symmetric strip of the width $\Delta$ located close to the separatrix striking point where the plasma pressure is the highest. However, the poloidal acceleration causes poloidal motion of the dust particle, which pushes it out of this region. Assuming that the acceleration acts along a distance of length 1 , we can estimate the magnitude of radial, $V_{d}^{(x)}$, and toroidal, $V_{d}^{(z)}$, velocity components that dust particle gains during the time it moves along that distance

$$
V_{d}^{(x)} \sim V_{d}^{(z)} \sim V_{d}^{(0)} \equiv V\left(\frac{\varsigma F M_{i} n_{s h}}{\tilde{\rho}_{d}} \frac{\eta}{r_{d}}\right)^{1 / 2} .
$$

For $\tilde{\rho}_{d} \sim 2 \mathrm{~g} / \mathrm{cm}^{3}, n_{s h}=3 \times 10^{13} \mathrm{~cm}^{-3}, T=10 \mathrm{eV}$, $\mathbb{Q} \sim 1 \mathrm{~cm}$, and $r_{d} \sim 3 \times 10^{-4} \mathrm{~cm}$ from Eq. (18) we find $V_{d}^{(0)} \sim 3 \times 10^{3} \mathrm{~cm} / \mathrm{s}$. This velocity is so large that if dust particle would move with such velocity toward the wall (as an example of such situation one can consider the motion of dust particle to the corner, see Fig. 6) it would penetrate through the sheath and hit the wall. Or, in case when dust particle is launched from the step (see Fig. 7), it can fly into the plasma volume at fairly large distance, $L_{\text {flight }}$. The length $L_{\text {flight }}$ depends on geometry of the launch, as well as on plasma flows in the regions far away from the sheath. We will discuss this aspect in the next Section. Here we shall only estimate the velocity of dust particle, $V_{w}$, that would allow dust particle to penetrate through the sheath barrier. We shall also estimate the distance along the wall, $\Delta_{w}$, along which velocity $V_{w}$ can be gained due to acceleration by plasma ions.

Since sheath potential drop is of the order of $T / \mathrm{e}$, in order to penetrate through the sheath the particle velocity $V_{d}$ and the length of trajectory $\Delta$ should satisfy inequality

$$
M_{d} V_{d}^{2}=2 \varsigma F n_{s h} T \pi r_{d}^{2} \Delta \tilde{>} 2 Z_{d} T .
$$

Then, taking into account Eq. (1), we find

$$
\begin{aligned}
& V_{d}>V_{w} \equiv V_{i}\left(\frac{4 \Lambda}{\varsigma_{F}} \frac{M_{i} n_{S h}}{\tilde{\rho}_{d}}\right)^{1 / 2} \frac{\lambda_{D}}{r_{d}} . \\
& E_{d} \equiv \frac{M_{d} V_{d}^{2}}{2} \tilde{>} E_{w} \equiv \Lambda r_{d}\left(\frac{T}{e}\right)^{2}, \\
& \Delta>\Delta_{w} \equiv \frac{4 \Lambda}{\varsigma_{F}} \frac{\lambda_{D}^{2}}{r_{d}} .
\end{aligned}
$$

For $n_{s h}=3 \times 10^{13} \mathrm{~cm}^{-3}, T=10 \mathrm{eV}, r_{d} \sim 3 \times 10^{-4} \mathrm{~cm}$, and $\tilde{\rho}_{d} \sim 2 \mathrm{~g} / \mathrm{cm}^{3}$, from the expressions (20)-(22) we find $V_{w} \approx 30 \mathrm{~cm} / \mathrm{s}, \quad E_{w} \approx 5 \times 10^{5} \mathrm{eV}, \quad$ and $\Delta_{w} \approx 10^{-3} \mathrm{~cm}$. We notice that for such plasma parameters it takes only about $10^{-4} s$ to accelerate dust particle to the speed $\sim V_{w}$.

As a result, the dust deposition areas on the wall structures can be far away from the location of dust origin.

\section{References}

1) P.K. Shukla and V.P. Silin, Physica Scripta 45 (1992) 508.

2) Y. Tomita , R. Smirnov, et al., to be published in J. Plasma and Fusion Res. SERIES, vol.6 (2004) .

3) J. E. Allen, Physica Scripta, 45 (1992) 497. 